\title{
The Concept of Lamina-Pedicle Perpendicularity: Part 1. Lumbar Spine
}

\author{
Tarek Anwar Elfiky ${ }^{1,}$, , Nirmal Dhananjay Patil ${ }^{2,}$, Keith DK Luk ${ }^{3}$, Mohamed Esam Faheem ${ }^{1}$, Dino Samartzis ${ }^{4}$ \\ ${ }^{1}$ Spine Unit, Al-Hadra University Hospital, Alexandria, Egypt \\ ${ }^{2}$ Department of Orthopaedics, Seth GS Medical College and KEM Hospital, Mumbai, India \\ ${ }^{3}$ Department of Orthopaedics \& Traumatology, Queen Mary Hospital, University of Hong Kong, Hong Kong \\ ${ }^{4}$ Department of Orthopaedic Surgery, Rush University Medical Center, Chicago, IL, USA
}

Study Design: Retrospective radiographic study.

Purpose: We hypothesized that the pedicle is almost perpendicular to the interlaminar line in the sagittal plane of the lumbar vertebrae. The current study aimed to define the lumbar lamina-pedicle inclination to verify the right-angle concept and to estimate the safety zones of sagittal inclination during pedicle screw insertion. To the best of our knowledge there are no previous similar studies. Overview of Literature: Based on our observations in different spinal disorders including deformities, we noted that following a sagittal (cranial-caudal) trajectory perpendicular to the interlaminar line joining the two adjacent vertebrae would work well in most of the vertebral levels.

Methods: This was a retrospective study on normal lumbar spine lateral radiographs of patients who presented with low back pain and were reviewed by two observers. Different inclination angles were constructed to estimate the safety zones of the pedicle screws' sagittal inclination.

Results: Radiographs of 30 consecutive patients, 25 females and five males, with a mean age of $39.43 \pm 11.18$ years, were studied. The mean angle of the interlaminar line and the pedicle axis was almost orthogonal at all the levels, with a range of $89.16^{\circ}-94.63^{\circ}$, which was not affected by the lumbar sagittal profile. The safety zones of the pedicle screws were measured, and they revealed a safe sagittal range of $19.73^{\circ}-24.40^{\circ}$ if the screw was inserted from the pedicle axis, $21.03^{\circ}-22.59^{\circ}$ if inserted from the most cephalic part, and $13.31^{\circ}-17.03^{\circ}$ if inserted from the most caudal part.

Conclusions: Our results confirmed the perpendicularity of the interlaminar line with the pedicle axis in the lumbar spine at all the levels. The interlaminar line is a useful guide for pedicle screw sagittal inclination.

Keywords: Lumbar; Lamina; Pedicle; Sagittal; Orthogonal

\section{Introduction}

Pedicle screw fixation is widely used in the treatment of deformities, fractures, and degenerative disorders [1-5].
Transpedicular instrumentation allows for three-dimensional fixation with a more rigid construct and has been shown to be clinically and biomechanically superior to hook rod constructs [6].

Received Apr 12, 2019; Revised Aug 30, 2019; Accepted Oct 13, 2019

Corresponding author: Tarek Anwar Elfiky

Spine Unit, Orthopedic Department, Al-Hadra University Hospital, Amprozo, Alexandria, Egypt

Tel: +20-1223382958, Fax: +20-25932907, E-mail: tarekfiky@yahoo.com

*These two authors contributed equally to this work. 
The unique neurologic and vascular anatomy near the pedicle makes optimal screw placement a critical issue. To minimize the risk of improper pedicle screw placement and to increase its safety and accuracy, various imageguided technologies, including the use of intraoperative fluoroscopy, intraoperative computed tomography (CT), and image-assisted navigation, have been developed [7-9]. However, such modalities may increase healthcare costs and radiation exposure to the patient, and the clinical efficacy compared to traditional techniques may be questionable [10]. The freehand technique of pedicle screw placement has been found to be accurate, reliable, and safe with low complication rates of and is potentially more cost-effective than image-guided techniques in treating a variety of spinal disorders [11].

While many studies have focused on screw inclination in the mediolateral direction [12-15], few have assessed the sagittal plane [16-19]. For proper sagittal trajectories of pedicle screws, bony landmarks, such as the lamina surface, the spinous process, and the facet joint tilt, can be used as anatomic references [20].

Based on our observations in different spinal disorders including deformities, we noted that following a sagittal (cranial-caudal) trajectory perpendicular to the interlaminar line joining the two adjacent vertebrae would work well in most of the vertebral levels. Since a long time, the senior author (K.D.L.) is using a simple technique in line with this concept by using a Langenbeck right-angle retractor intraoperatively. In this method, the short limb of the retractor was placed over the ipsilateral or contralateral posterior aspect of the inferior edges of the lamina to be screwed and the one above, and the inclination of the long limb was followed during insertion of pedicle screws to determine the sagittal inclination. This technique was used for placement of lumbar as well as thoracic screws (Fig. 1A, B). However, to date there is no clear literature in support of this method, and there is no consensus on the definition of the sagittal trajectory of pedicle screw insertion.

Our hypothesis was that the pedicle axis is almost perpendicular to the interlaminar line in the lumbar vertebra. The objective of the current study was to define the sagittal lamina-pedicle inclination and to estimate the safety zones for pedicle screw insertion. To the best of our knowledge, there are no previous similar studies.

\section{Materials and Methods}

\section{Participants}

This was a retrospective study on lumbar spine supine lateral radiographs of patients who presented to Spine Surgery Unit, Department of Orthopedics and Traumatology, Alexandria University with low back pain between January 2017 and December 2017. Normal standing radiographs were selected by two spine consultants at our institute. The study protocol was approved by the Institutional Review Board and Ethics Committee (IRB approval

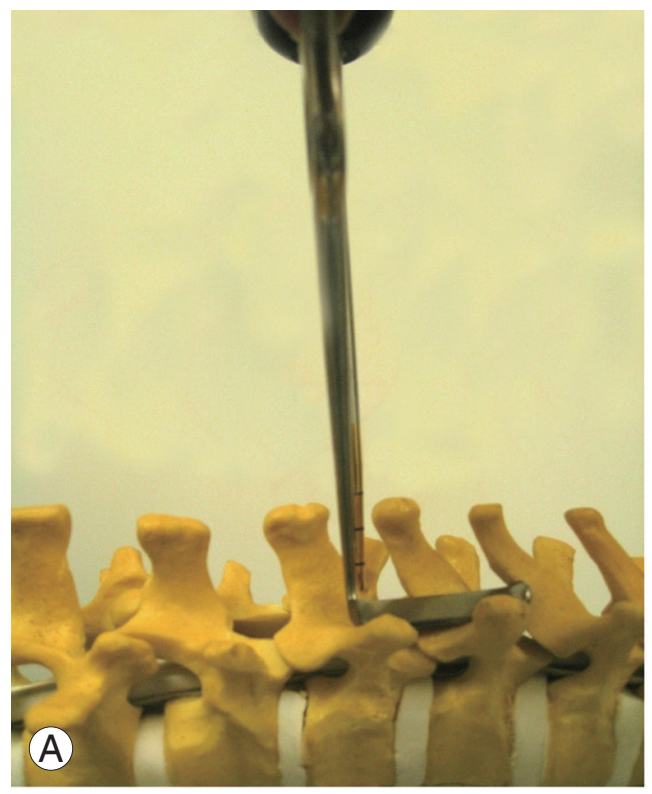

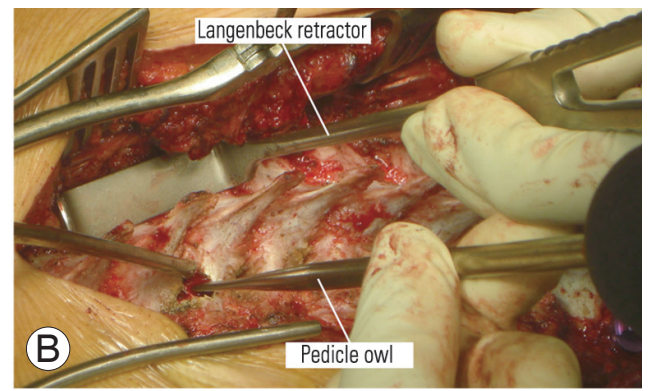

Fig. 1. (A) Anatomical model showing the sagittal profile of the pedicle screw inclination in the vertebra. If the short limb of a Langenbeck right-angle retractor is placed over the ipsilateral or contralateral posterior aspect of the inferior edges of the lamina to be screwed and the one above, the sagittal inclination may be determined by following the inclination of the long limb of the retractor during insertion of pedicle screws. This technique was used for placement of the lumbar as well as the thoracic screws. (B) Intraoperative demonstration of the technique for determining the inclination of the pedicle screw. 
no., 129-15). Informed consent was obtained from each patient.

The exclusion criteria were as follows: (1) radiographs with fractures, tumors, infections, and significant degenerative changes in the spine; (2) previous surgery; and (3) underexposed radiographs.

\section{Method of measurement}

All images were independently reviewed by two independent observers. Both were spine fellows with special experience in spinal deformities. Before measurements were taken, there was an agreement between the observers to draw the interlaminar line and the pedicle axis for each level. The interlaminar line was defined as the line connecting the two adjacent laminae, joining the lower edge of the lamina above to the lower edge of the lamina of the measured vertebra.

\section{Definitions}

\section{1) Lines (Fig. 2A)}

Line a (interlaminar line): the tangential line between the dorsal edge of the caudal end of the lamina of the same level and the one cranial to it. Line b: the line of the inner superior border of the pedicle wall and parallel to the upper end plate of the vertebrae. Line c: a line of the inner inferior border of the pedicle wall and parallel to the upper end plate. Line $d$ (pedicle axis): a line midway between line $\mathrm{b}$ and line $\mathrm{c}$.

\section{2) The measurements to be taken}

The measurements to be taken were as follows: interlaminar line-pedicle axis angle: the angle between line a and line d (Fig. 2B); inferior safety angle (Fig. 2C); superior safety angle (Fig. 2D); and safety angle along the pedicle axis (Fig. 2E).

Inferior safety angle, superior safety angle, and safety angle along the pedicle axis were used to determine the cephalic as well as the caudal safety zone limit for pedicle screw insertion without violation of either of the pedicular borders.

\section{3) Vertebral zones (Fig. 2F)}

Zone A: upper third of the anterior vertebral border. Zone $\mathrm{B}$ : middle third of the anterior vertebral border. Zone C: lower third of the anterior vertebral border. In addition, the lumbar sagittal profile was measured between the upper border of L1 and the upper border of S1.
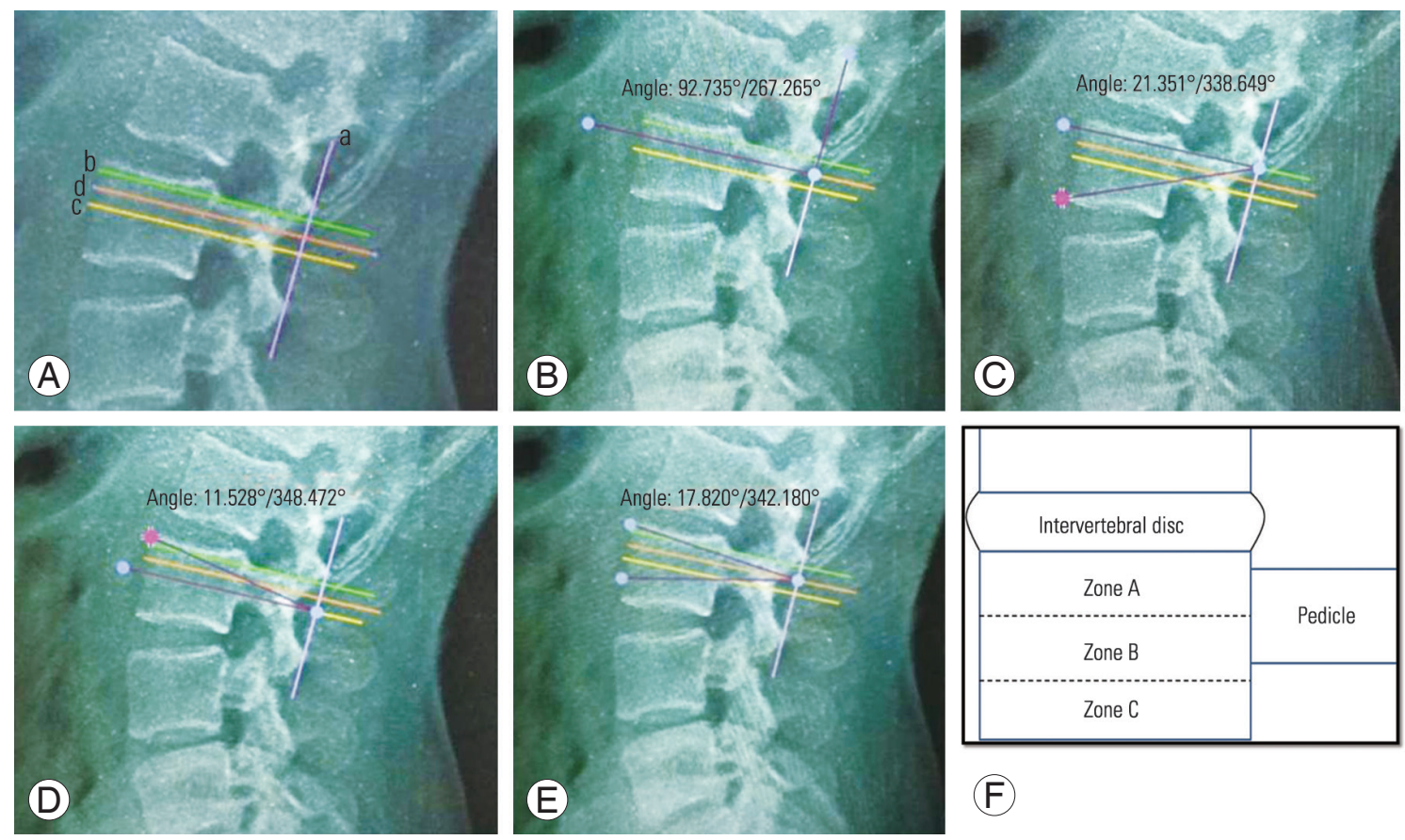

Fig. 2. (A) Definition of lines. (B) Pedicle axis interlaminar angle. (C) Inferior safety angle. (D) Superior safety angle. (E) Safety angle along the pedicle axis. (F) Vertebral body zones. 


\section{Statistical analysis}

Descriptive statistics were used for parameters which did not need statistical analysis. Correlations between parameters were assessed using a Pearson's correlation test. The interclass correlation coefficient (ICC) was used to determine the interobserver reliability. Microsoft Office (Microsoft Corp., Redmond, WA, USA), SPSS software ver. 9.4 (SPSS Inc., Chicago, IL, USA), and GraphPad software (GraphPad Software, San Diego, CA, USA) were used for the analysis.

\section{Results}

The study material included plain radiographs of 30 consecutive patients. There were 25 females and five males with a mean age of $39.43 \pm 11.18$ years (range, 25-72 years). The interobserver reliability was excellent (ICC $=0.9)$. Therefore, the mean of the values recorded by the two observers was used for each patient. The mean lumbar lordosis was $56.69^{\circ} \pm 13.81^{\circ}$.

\section{Interlaminar line-Pedicle axis angle}

As illustrated in Table 1, the mean interlaminar line-

\begin{tabular}{|c|c|}
\hline Vertebrae & ne-pedicle axis angle $\left({ }^{\circ}\right)$ \\
\hline D12 & $89.54 \pm 2.17$ \\
\hline L1 & $90.86 \pm 1.6$ \\
\hline L2 & $89.45 \pm 2.22$ \\
\hline L3 & $89.16 \pm 2.18$ \\
\hline L4 & $90.27 \pm 2.14$ \\
\hline L5 & $94.63 \pm 3.84$ \\
\hline
\end{tabular}

Values are presented as mean \pm standard deviation.

Table 2. Safety sagittal angle along pedicle axis

\begin{tabular}{lc} 
Vertebrae & Safety sagittal angle along pedicle axis $\left({ }^{\circ}\right)$ \\
\hline D12 & $23.69 \pm 2.86$ \\
L1 & $23.54 \pm 2.80$ \\
L2 & $21.65 \pm 3.20$ \\
L3 & $19.73 \pm 3.13$ \\
\hline L4 & $20.08 \pm 3.20$ \\
\hline L5 & $24.40 \pm 3.24$ \\
\hline
\end{tabular}

Values are presented as mean \pm standard deviation. pedicle axis angle was almost orthogonal for all the levels. It ranged from $89.16^{\circ} \pm 2.18^{\circ}$ at L3 to $94.63^{\circ} \pm 3.84^{\circ}$ at L5.

\section{Safe sagittal angle along pedicle axis}

Table 2 shows the mean safe sagittal angle along the pedicle axis for all levels before violation of either the cephalic or caudal limits of the pedicle. The mean values ranged from $19.73^{\circ} \pm 3.13^{\circ}$ at L3 to $24.40^{\circ} \pm 3.24^{\circ}$ at L5.

\section{Inferior safe sagittal angle along the superior pedicle}

Table 3 demonstrates the mean safety sagittal angle along the superior pedicle before violation of the caudal limit of the pedicle. The values were similar to the safe angle along the pedicle axis, ranging between $21.03^{\circ} \pm 2.87^{\circ}$ and $22.59^{\circ} \pm 2.10^{\circ}$

\section{Superior safe sagittal angle along inferior pedicle}

Table 4 demonstrates the mean safety sagittal angle along the superior pedicle before violation of the cephalic limit of the pedicle. It was less than the values along the axis or superior pedicle and ranged from $13.31^{\circ} \pm 1.72^{\circ}$ at $\mathrm{L} 3$ to $17.03^{\circ} \pm 2.69^{\circ}$

Table 3. Safety sagittal angle along superior pedicle Vertebrae Safety sagittal angle along superior pedicle $\left({ }^{\circ}\right)$

\begin{tabular}{ll} 
D12 & $21.13 \pm 2.02$ \\
L1 & $21.52 \pm 2.46$ \\
L2 & $22.59 \pm 2.10$ \\
L3 & $21.92 \pm 2.14$ \\
L4 & $21.03 \pm 2.87$ \\
L5 & $21.48 \pm 2.67$ \\
\hline
\end{tabular}

Values are presented as mean \pm standard deviation.

Table 4. Safety sagittal angle along inferior pedicle

Vertebrae Safety sagittal angle along inferior pedicle $\left({ }^{\circ}\right)$

\begin{tabular}{ll} 
D12 & $15.72 \pm 2.19$ \\
\hline L1 & $16.25 \pm 2.02$ \\
L2 & $13.97 \pm 3.05$ \\
L3 & $13.31 \pm 1.72$ \\
L4 & $13.86 \pm 2.66$ \\
\hline L5 & $17.03 \pm 2.69$ \\
\hline
\end{tabular}

Values are presented as mean \pm standard deviation. 


\section{Vertebral zone of exit of the perpendicular line}

Table 5 and Fig. 3 show that the line perpendicular to the interlaminar line fell mostly into zone A. In no cases did the line fall into zone $\mathrm{C}$. This finding was highly statistically significant $(p<0.0001)$.

\section{Correlation between lumbar lordosis and interlami- nar line-pedicle axis angle in various vertebrae}

There was no correlation between lumbar lordosis and the interlaminar line-pedicle axis angle, implying that the

\begin{tabular}{lccc}
\multirow{2}{*}{$\begin{array}{l}\text { Table 5. Vertebral zone of exit of the perpendicular line } \\
\text { Vertebrae }\end{array}$} & \multicolumn{2}{c}{$\begin{array}{c}\text { Zone of exit } \\
\text { A-value }\end{array}$} \\
\cline { 2 - 3 } & A & B & \\
\hline D12 & $27(90.00)$ & $3(10.00)$ & $<0.0001$ \\
L1 & $29(96.67)$ & $1(3.33)$ & $<0.0001$ \\
\hline L2 & $25(83.33)$ & $5(16.67)$ & $<0.0001$ \\
L3 & $27(90.00)$ & $3(10.00)$ & $<0.0001$ \\
L4 & $28(93.33)$ & $2(6.67)$ & $<0.0001$ \\
L5 & $28(93.33)$ & $2(6.67)$ & $<0.0001$ \\
\hline
\end{tabular}

Values are presented as number (\%).

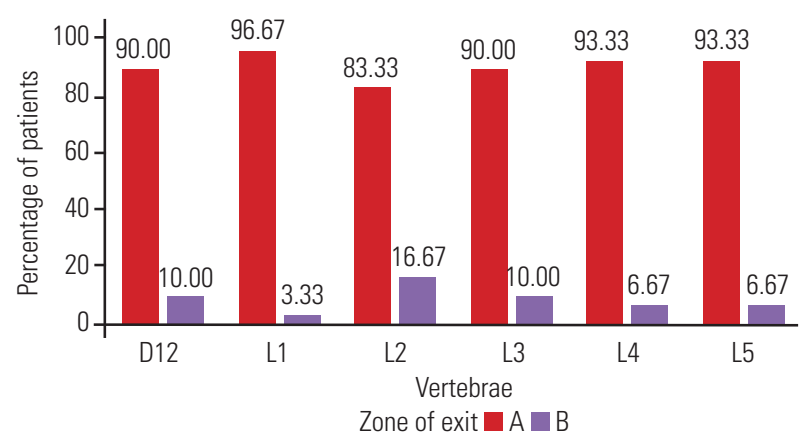

Fig. 3. Vertebral zone of exit of the perpendicular line.

Table 6. Correlation between lumbar lordosis and interlaminar line-pedicle axis angle in the various vertebrae

\begin{tabular}{lcc} 
Vertebrae & $r$ & $p$-value \\
D12 & -0.1062 & 0.5772 \\
L1 & -0.088 & 0.6438 \\
L2 & -0.3593 & 0.0514 \\
L3 & -0.3464 & 0.0611 \\
L4 & -0.0189 & 0.9248 \\
L5 & -0.0387 & 0.8420 \\
\hline
\end{tabular}

orthogonal concept is not affected by the lumbar sagittal profile and that even for patient with different lordosis, the pedicle axis maintains a constant relationship with the interlaminar line (Table 6).

\section{Discussion}

Our objective was to prove perpendicularity of the pedicle axis to the interlaminar line, the traditional concept upon which many spine surgeons depend in pedicle screw sagittal inclination. An additional objective was to assess the safe sagittal inclination of the pedicle screw before cephalic and caudal pedicle breach (sagittal safety zone), as such a breach may lead to injury to the nerves roots, an intradiscal screw placement, or loss of purchase of the screw. T12 was also assessed because of its simplicity on plain radiographs.

Our results confirmed the perpendicularity in the lumbar spine at all the vertebral levels (T12-L5). This has important surgical applications, facilitating intraoperative sagittal inclination of lumbar pedicle screws in freehand placement.

Although we found no similar studies concerning the lumbar spine, few reported that the freehand sagittal trajectory was orthogonal to the dorsal spine curvature. Fennell et al. [16] used the orthogonal sagittal inclication in 33 patients. However, detailed measurements of the individual vertebrae or identification of vertebral zones were not carried out. Furthermore, Oshina et al. [20] reported that the ideal sagittal trajectories for pedicle screw insertions were nearly orthogonal to the lamina surface or to the line connecting spinous processes, but were different for each vertebra, although the lamina surface method was the most reliable for the C7-T1 vertebrae. However, their series was limited to pedicle screws between $\mathrm{C} 7$ and T1 [20]. In addition, Rivkin et al. [21] reported that the sagittal trajectory is perpendicular to the long axis of the lamina. However, their study was restricted to $\mathrm{T} 1$ pedicle screws.

An additional finding in our results was that the angle of inclination was highest at L5, with a mean of $94.63^{\circ} \pm 3.84^{\circ}$ but was still almost orthogonal. This may be explained by the pedicle morphology. Cephalocaudal inclination increased significantly towards the lower levels of the lumbar spine below L3, with a maximum at L5 [22].

Interestingly, majority of the perpendicular lines in our series were positioned in zone A (located adjacent to the 
superior endplate) and none in zone C. Previous biomechanical studies supported the superiority of the straightforward technique over the anatomic trajectory technique [23]. Cho et al. [24] reported that a trajectory parallel or caudal to the superior endplate can minimize screw breakage from repeated axial loading, and that straight insertion of the pedicle screw in the mid-sagittal plane provides the strongest stability. We believe that the superior mechanical property of the straightforward technique was due to the fact that the screw engages more of the superior cortex of the pedicle as well as the compact cancellous bone along the superior endplate of the vertebral body. However, it should be noted that several other factors can affect the pedicle screw stability, including the sagittal and mediolateral inclination, the depth of screw insertion, and bone quality [23].

An additional important finding in our series was that the orthogonal relationship was not affected by the lumbar sagittal profile. Even for patients with different lordoses, the pedicle axis maintains a constant relationship with the interlaminar line. This means that surgeons should use the interlaminar line as a reference and should not depend on predefined sagittal angulation on a lateral radiograph. This is especially true in the lumbar spine because of its mobility.

The concept of the mediolateral pedicle screws' "safe zone" has been previously addressed [11]. However, we are unaware of any previous studies concerned with the sagittal safe zone. To assess the sagittal safe zone, we measured three different angles: safe angle along the pedicle axis, and both superior and inferior safe angles, to estimate the safety of sagittal pedicle screw inclination when inserted into the middle and the most cephalad and caudal parts of the pedicle. Our results demonstrated a safe range of $19.73^{\circ}-24.40^{\circ}$ if the screw is inserted along its axis, or $21.03^{\circ}-22.59^{\circ}$ if inserted along the most cephalic part. The least safe was when the screw was placed along the most caudal part of the pedicle, with a range of $13.31^{\circ}-17.03^{\circ}$. This means that the safety zone is the smallest if the screw is inserted from the most caudal part of the pedicle and directed cranially.

It should be noted that we do not recommend any new entry point for this technique. The ideal entry point is along the pedicle axis (at the junction of lateral border of superior facet and line running through the upper third of the transverse process). As such, the screw can be inserted perpendicular to the interlaminar line. However, if the entry point is superior or inferior, the angulation would change accordingly. With a superior entry point, the angulation will be less than $90^{\circ}$ and directed caudally, whereas with an inferior entry point, the angulation will be more than $90^{\circ}$ and directed cranially to avoid violation of the pedicle walls. The distance between the superior and inferior entry point on the posterior surface is equal to the height of the pedicle on lateral view with the central point being the one along the pedicle axis. The main aim of defining the safe angle of pedicle screw insertion was to determine the range of play of angles possible with an entry point superior or inferior to the traditional entry point along the pedicle axis and along the superior or the inferior border of the pedicle, respectively.

The information gained from the current study will be useful in the open technique of lumbar pedicle screw sagittal inclination by improving understanding of the direction in which pedicle screws should be inserted. Inserting screws in the lumbar spine is usually straightforward, but it can be more difficult in deformities (e.g., kyphosis). Many novice surgeons often use repeated lateral C-arm shoots to guide the sagittal trajectory of screws. This technique gives the surgeon a good visual cue and can limit
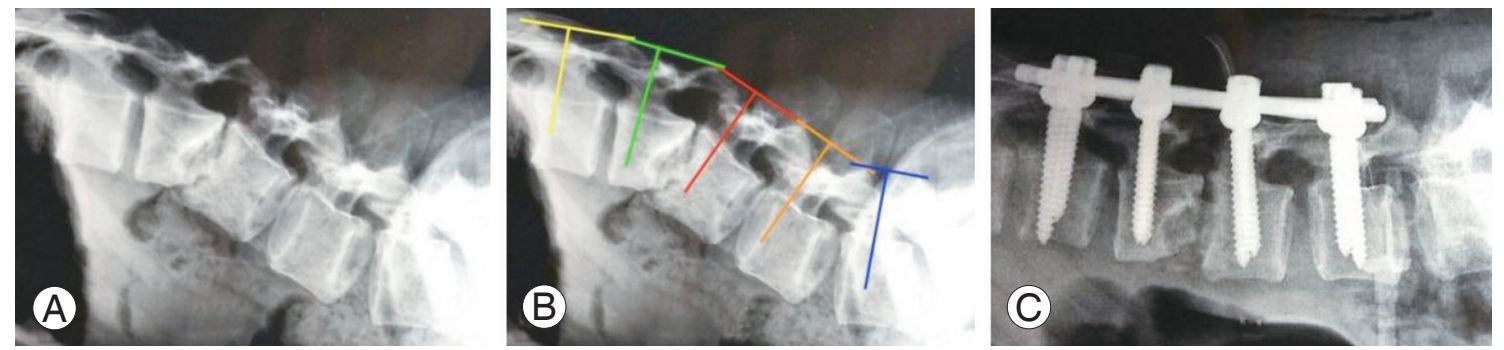

Fig. 4. (A) A radiograph simulating intraoperative prone position of a case of $L 2-L 3$ spondylodiscitis. The sagittal profile changes proximal to the lesion. (B) Interlaminar line and a line perpendicular to the interlaminar line passing through the base of the superior articular facet are drawn for each level (simulating the short limb and the long limb of the right-angled instrument). The screw exit was in the safe zone A and B of the respective levels. (C) Postoperative radiograph of the same patient. 
the use of fluoroscopy and exposure to ionizing radiation (Fig. 4). Although the method described in the present study is useful in open techniques, it may not be useful or applicable in percutaneous image-guided methods.

Perpendicular screw placement is a matter of surgical experience. Stanescu et al measured the angle between the posterior aspect of the lamina and the pedicle axis by placing one arm of a goniometer on the posterior aspect of the lamina [19]. We suggest using any right-angle instrument, such as a right-angle Kocher-Langenbeck retractor, with the short arm placed over the posteroinferior edges of the two adjacent laminae. The sagittal inclination of the long limb of the retractor will serve as a guide to the craniocaudal trajectory of the screw. This technique is novel, unique, simple, with decreased human errors, and has been used in the lumbar and thoracic vertebrae in various spinal disorders, including deformities. However, it is not yet published. Our experimental model, as shown in Fig. 1 , has proved the efficacy of this method. It should also be noted that there are situations in which the interlaminar line does not work or needs modification. When there is a spondylolisthesis, one should not use the 'slipped laminae' for the interlaminar line. Instead, one should use the two laminae above or below the slip.

There are few limitations to the current study. First, the study would have more power and reliability if the sample size was expanded. Second, there is a lack of intraobserver assessment. Finally, the mediolateral inclination, which is usually the more difficult part, was beyond the scope of the current study.

\section{Conclusions}

Based on our results, we support the concept of lamina pedicle perpendicularity in the lumbar spine at all vertebral levels (T12-L5), with slightly more caudal inclination at L5. The lamina is a useful intraoperative guide for pedicle screw sagittal inclination.

The lamina-pedicle orthogonal relation is not affected by the lumbar sagittal profile, and even for patients with different sagittal profiles, the pedicle maintains a constant relationship with the interlaminar line. The possible safety zones for sagittal pedicle screw have been demonstrated using three different points in relation to the pedicle, and the findings showed that the smallest sagittal safety zone was found when we insert screws from the most caudal part of the pedicle.

\section{Conflict of Interest}

No potential conflict of interest relevant to this article was reported.

\section{Author Contributions}

Study design: TE, NDP; writing the manuscript: TE; radiographic assessment: NDP, MEF; statistical analysis: NDP; provision of the concept, the data interpretation, and critical review: KDL; and manuscript preparation and critical review: DS.

\section{References}

1. Delorme S, Labelle H, Aubin CE, et al. A threedimensional radiographic comparison of CotrelDubousset and Colorado instrumentations for the correction of idiopathic scoliosis. Spine (Phila Pa 1976) 2000;25:205-10.

2. Suk SI, Lee CK, Min HJ, Cho KH, Oh JH. Comparison of Cotrel-Dubousset pedicle screws and hooks in the treatment of idiopathic scoliosis. Int Orthop 1994;18:341-6.

3. Upendra BN, Meena D, Chowdhury B, Ahmad A, Jayaswal A. Outcome-based classification for assessment of thoracic pedicular screw placement. Spine (Phila Pa 1976) 2008;33:384-90.

4. Yuan HA, Garfin SR, Dickman CA, Mardjetko SM. A historical cohort study of pedicle screw fixation in thoracic, lumbar, and sacral spinal fusions. Spine (Phila Pa 1976) 1994;19(20 Suppl):2279S-96S.

5. Gaines RW Jr. The use of pedicle-screw internal fixation for the operative treatment of spinal disorders. J Bone Joint Surg Am 2000;82:1458-76.

6. Kim YJ, Lenke LG, Cho SK, Bridwell KH, Sides B, Blanke K. Comparative analysis of pedicle screw versus hook instrumentation in posterior spinal fusion of adolescent idiopathic scoliosis. Spine (Phila Pa 1976) 2004;29:2040-8.

7. Carbone JJ, Tortolani PJ, Quartararo LG. Fluoroscopically assisted pedicle screw fixation for thoracic and thoracolumbar injuries: technique and short-term complications. Spine (Phila Pa 1976) 2003;28:91-7.

8. Cui G, Wang Y, Kao TH, et al. Application of intraoperative computed tomography with or without navigation system in surgical correction of spinal 
deformity: a preliminary result of 59 consecutive human cases. Spine (Phila Pa 1976) 2012;37:891-900.

9. Zhang C, Wang Z, Zhang C, Chen F, Zhang H, Yan X. Spine Bull's-Eye Robot guidewire placement with pedicle standard axis view for thoracic and lumbar pedicle screw fixation. J Spinal Disord Tech 2012;25:E191-8.

10. Kassis SZ, Abukwedar LK, Msaddi AK, Majer CN, Othman W. Combining pedicle screw stimulation with spinal navigation, a protocol to maximize the safety of neural elements and minimize radiation exposure in thoracolumbar spine instrumentation. Eur Spine J 2016;25:1724-8.

11. Kim YW, Lenke LG, Kim YJ, et al. Free-hand pedicle screw placement during revision spinal surgery: analysis of 552 screws. Spine (Phila Pa 1976) 2008;33:1141-8.

12. Liljenqvist U, Hackenberg L, Link T, Halm H. Pullout strength of pedicle screws versus pedicle and laminar hooks in the thoracic spine. Acta Orthop Belg 2001;67:157-63.

13. Luhmann SJ, Lenke LG, Kim YJ, Bridwell KH, Schootman M. Thoracic adolescent idiopathic scoliosis curves between 70 degrees and 100 degrees: is anterior release necessary? Spine (Phila $\mathrm{Pa} 1976$ ) 2005;30:2061-7.

14. Roy-Camille R, Saillant G, Mazel C. Plating of thoracic, thoracolumbar, and lumbar injuries with pedicle screw plates. Orthop Clin North Am 1986;17:14759.

15. Halm H, Niemeyer T, Link T, Liljenqvist U. Segmental pedicle screw instrumentation in idiopathic thoracolumbar and lumbar scoliosis. Eur Spine J 2000;9:191-7.

16. Fennell VS, Palejwala S, Skoch J, Stidd DA, Baaj AA. Freehand thoracic pedicle screw technique using a uniform entry point and sagittal trajectory for all levels: preliminary clinical experience. J Neurosurg Spine 2014;21:778-84.

17. Li J, Zhao $\mathrm{H}$, Xie $\mathrm{H}$, et al. A new free-hand pedicle screw placement technique with reference to the supraspinal ligament. J Biomed Res 2014;28:64-70.

18. Parker SL, McGirt MJ, Farber SH, et al. Accuracy of free-hand pedicle screws in the thoracic and lumbar spine: analysis of 6816 consecutive screws. Neurosurgery 2011;68:170-8.

19. Stanescu S, Ebraheim NA, Yeasting R, Bailey AS, Jackson WT. Morphometric evaluation of the cervico-thoracic junction: practical considerations for posterior fixation of the spine. Spine (Phila Pa 1976) 1994;19:2082-8.

20. Oshina M, Horii C, Hirai S, et al. Comparison of freehand sagittal trajectories for inserting pedicle screws between C7 and T5. Clin Spine Surg 2018;31:E35762.

21. Rivkin MA, Okun JF, Yocom SS. Novel free-hand T1 pedicle screw method: review of 44 consecutive cases. J Neurosci Rural Pract 2014;5:349-54.

22. Makino T, Kaito T, Fujiwara H, Yonenobu K. Analysis of lumbar pedicle morphology in degenerative spines using multiplanar reconstruction computed tomography: what can be the reliable index for optimal pedicle screw diameter? Eur Spine J 2012;21:1516-21.

23. Lehman RA Jr, Polly DW Jr, Kuklo TR, Cunningham B, Kirk KL, Belmont PJ Jr. Straight-forward versus anatomic trajectory technique of thoracic pedicle screw fixation: a biomechanical analysis. Spine (Phila Pa 1976) 2003;28:2058-65.

24. Cho W, Cho SK, Wu C. The biomechanics of pedicle screw-based instrumentation. J Bone Joint Surg Br 2010;92:1061-5. 